\title{
Classical Consequences of Continuous Choice Principles from Intuitionistic Analysis
}

\author{
François G. Dorais
}

\begin{abstract}
The sequential form of a statement

$$
\forall \xi(B(\xi) \rightarrow \exists \zeta A(\xi, \zeta))
$$

is the statement$$
\forall \xi\left(\forall n B\left(\xi_{n}\right) \rightarrow \exists \zeta \forall n A\left(\xi_{n}, \zeta_{n}\right)\right) .
$$

There are many classically true statements of the form $(\dagger)$ whose proofs lack uniformity, and therefore the corresponding sequential form is not provable in weak classical systems. The main culprit for this lack of uniformity is of course the law of excluded middle. Continuing along the lines of Hirst and Mummert, we show that if a statement of the form $(\dagger)$ satisfying certain syntactic requirements is provable in some weak intuitionistic system, then the proof is necessarily sufficiently uniform that the corresponding sequential form is provable in a corresponding weak classical system. Our results depend on Kleene's realizability with functions and the Lifschitz variant thereof.
\end{abstract}

\section{Introduction}

In [1], Brouwer introduced the continuity theorem, which states that every function on the unit interval is (uniformly) continuous. While many other principles of intuitionistic analysis are classically valid (e.g., the fan theorem and the bar theorem), Brouwer's continuity theorem contradicts the law of excluded middle. Indeed, were the equality of two real numbers decidable, then the characteristic function of the singleton $\{0\}$ would be an example of a discontinuous function defined on the unit interval.

Still, many formal systems of constructive analysis either satisfy Brouwer's continuity theorem or are compatible with it. In fact, variants of the continuity theorem

Received February 27, 2011; accepted July 28, 2012

2010 Mathematics Subject Classification: Primary 03B20; Secondary 03B30, 03F35, 03F55

Keywords: intuitionistic analysis, second-order arithmetic, reverse mathematics, realizability, choice principles

(C) 2014 by University of Notre Dame $\quad$ 10.1215/00294527-2377860 
are often combined with the (classically valid) choice principles to yield continuous choice principles of the following form:

If for every $\xi$ there is a $\zeta$ such that $A(\xi, \zeta)$, then there is a continuous function $F$ such that $A(\xi, F(\xi))$ holds for all $\xi$.

When $\xi$ and $\zeta$ are interpreted as varying over the unit interval (or the real numbers or Cantor space or Baire space), this enforces a highly constructive strength to the existential quantifier. Indeed, the continuity of $F$ allows us to effectively translate finitary information about the argument $\xi$ into finitary information about a witness $\zeta$ to the statement $A(\xi, \zeta)$. Thus, even in very weak systems where infinitary constructions are hardly formalizable, one can still use $F$ to simultaneously transform an infinite sequence of arguments $\left\langle\xi_{0}, \xi_{1}, \ldots\right\rangle$ into a corresponding sequence of witnesses $\left\langle\zeta_{0}, \zeta_{1}, \ldots\right\rangle$ such that $A\left(\xi_{n}, \zeta_{n}\right)$ holds for every $n$.

This general idea was exploited by Hirst and Mummert [4] to show that if $A(\xi, \zeta)$ has a special syntactic form, then

$$
\mathrm{E}-\mathrm{HA}{ }^{\omega}+\mathrm{AC} \vdash \forall \xi \exists \zeta A(\xi, \zeta)
$$

implies

$$
\mathrm{RCA}^{\omega} \vdash \forall \xi \exists \zeta \forall n A\left(\xi_{n}, \zeta_{n}\right),
$$

where $E-H A^{\omega}$ is a system Heyting arithmetic with extensional higher types that is used in proof theory (cf. Kohlenbach [7]), AC is the full axiom of choice, and RCA ${ }^{\omega}$ is a variant with higher types of Friedman's classical system of recursive comprehension that is used in reverse mathematics (cf. Kohlenbach [6]).

The results of Hirst and Mummert are based on Kreisel's modified realizability and Gödel's Dialectica interpretation. In this paper, we use Kleene's realizability with functions and a Lifschitz variant thereof due to van Oosten [10] to obtain similar results. Our first result (Corollary 3.9) shows in particular that if $A(\xi, \zeta)$ satisfies certain syntactic requirements, then

$$
\mathrm{EL}+\mathrm{GC} \vdash \forall \xi \exists \zeta A(\xi, \zeta),
$$

implies

$$
\mathrm{RCA} \vdash \forall \xi \exists \zeta \forall n A\left(\xi_{n}, \zeta_{n}\right),
$$

where EL is a system of intuitionistic analysis described in the next section and GC is a strong continuous choice principle that implies Brouwer's continuity theorem. Our second result (Corollary 4.9) is similar except that it incorporates the weak König lemma (WKL). If $A(\xi, \zeta)$ satisfies certain syntactic requirements, then

$$
\mathrm{EL}+\mathrm{WKL}+\mathrm{GC}_{L} \vdash \forall \xi \exists \zeta A(\xi, \zeta)
$$

implies

$$
\mathrm{RCA}+\mathrm{WKL} \vdash \forall \xi \exists \zeta \forall n A\left(\xi_{n}, \zeta_{n}\right)
$$

where $\mathrm{GC}_{L}$ is a weakening of GC that does not imply continuous choice but still implies Brouwer's continuity theorem. This result is very interesting since WKL is not generally recognized as a constructive principle.

\section{The Systems EL and RCA}

Our base system for intuitionistic analysis is a minor variant of the system EL described by Troelstra [9, Section 1.9.10]. This is a system with two sorts: numbers and (unary) functions. We will generally use roman letters $a, b, c, \ldots$ to range over number terms and Greek letters $\alpha, \beta, \gamma, \ldots$ to range over function terms. The terms 
of the language are built as follows:

- number variables are number terms;

- function variables are function terms;

- the zero constant 0 is a number term;

- the successor constant $\sigma$ is a function term;

- if $t_{1}, \ldots, t_{k}$ are number terms and $f$ is a symbol for a $k$-ary primitive recursive function, then $f\left(t_{1}, \ldots, t_{k}\right)$ is a number term;

- if $t$ is a number term and $\tau$ is a function term, then the evaluation $\tau(t)$ is a number term;

- if $t$ is a number term and $x$ is a number variable, then $\lambda x$. $t$ is a function term;

- if $t$ is a number term and $\tau$ is a function term, then $\operatorname{R} t \tau$ is a function term.

The only atomic relation in our language is equality for the number sort; equality for the function sort is defined by extensionality:

$$
\alpha=\beta \leftrightarrow \forall x(\alpha(x)=\beta(x)) .
$$

Formulas are built in the usual way for intuitionistic systems, except that we think of the disjunction $A \vee B$ as an abbreviation for

$$
\exists x((x=0 \rightarrow A) \wedge(x \neq 0 \rightarrow B)) .
$$

Since equality for the number sort is decidable, this is equivalent to the usual intuitionistic disjunction (see [9, Section 1.3.7]).

In addition to the usual intuitionistic logic axioms, our base systems have the usual equality axioms and the defining axioms for all primitive recursive functions. Of course, for this to make sense, the zero and successor constants must satisfy

$$
\sigma(x) \neq 0 \wedge(\sigma(x)=\sigma(y) \rightarrow x=y)
$$

and the induction scheme

$$
\forall x(A(x) \rightarrow A(\sigma(x))) \rightarrow \forall x(A(0) \rightarrow A(x)),
$$

where $A(x)$ is any formula. The last two term formation rules are governed by the $\lambda$-conversion scheme

$$
(\lambda x . t)\left(t^{\prime}\right)=t\left[x / t^{\prime}\right]
$$

and the recursion scheme

$$
(\mathrm{R} t \tau)(0)=t \wedge(\mathrm{R} t \tau)\left(\sigma\left(t^{\prime}\right)\right)=\tau\left((\mathrm{R} t \tau)\left(t^{\prime}\right)\right) .
$$

Moreover, we have the following choice scheme:

$$
\forall x \exists y A(x, y) \rightarrow \exists \alpha \forall x A(x, \alpha(x)),
$$

where $A(x, y)$ is a quantifier-free formula. The system $\mathrm{EL}_{0}$ is defined in exactly the same way, except that IA is replaced by the quantifier-free induction axiom QF-IA.

Although not part of our base systems, we will often make use of the Markov principle

$$
\neg \neg \exists x(\alpha(x)=0) \rightarrow \exists x(\alpha(x)=0) .
$$

This principle is a simple consequence of the law of excluded middle (LEM), which distinguishes classical systems from intuitionistic systems. We define RCA and $\mathrm{RCA}_{0}$ to be the classical systems EL $+\mathrm{LEM}$ and $\mathrm{EL}_{0}+\mathrm{LEM}$, respectively. These are function-based systems which are equivalent to the set-based system of recursive comprehension (with full induction and just $\Sigma_{1}^{0}$-induction, respectively) traditionally used in reverse mathematics (see Simpson [8]). 
Since our basic systems have symbols for all primitive recursive functions, pairs and sequences of numbers can be encoded in the usual manner. The length of a finite sequence $x$ is denoted by $|x|$. We write $\left\langle x_{0}, \ldots, x_{n-1}\right\rangle$ for the finite sequence of length $n$ whose $(i+1)$ th term is $x_{i}$. The concatenation of $x$ and $y$ is denoted $x^{\wedge} y$. We will often view functions as infinite sequences of numbers. If $\alpha$ is a function, we write $\bar{\alpha} n$ for the finite initial segment $\langle\alpha(0), \ldots, \alpha(n-1)\rangle$.

For pairs and sequences of functions, we use the following encoding schemes. Define

$$
\pi_{0}=\lambda n .2 n, \quad \pi_{1}=\lambda n \cdot 2 n+1 .
$$

If $\alpha, \beta$ are two functions, then $\langle\alpha, \beta\rangle$ denotes the unique function such that $\langle\alpha, \beta\rangle \pi_{0}=\alpha$ and $\langle\alpha, \beta\rangle \pi_{1}=\beta$. In a similar fashion, any function $\alpha$ can also be viewed as an infinite sequence of functions where the $(m+1)$ th such function is

$$
\alpha_{m}=\lambda n \cdot \alpha\left(2^{m}(2 n+1)-1\right) .
$$

When it makes sense, we will write $\left\langle\alpha_{m}\right\rangle_{m=0}^{\infty}$ for the unique $\alpha$ whose $(m+1)$ th component is $\alpha_{m}$. Number-function pairs are encoded by concatenation; that is, $\langle n\rangle^{\wedge} \alpha$ denotes the unique function such that $\left(\langle n\rangle^{\wedge} \alpha\right)(0)=n$ and $\left(\langle n\rangle^{\wedge} \alpha\right) \sigma=\alpha$.

2.1 Kleene's second algebra in EL Our results of Section 3 depend on Kleene's realizability with functions. The base system EL is tailored to formalize this notion of realizability. To do this, we need to discuss the representation of partial continuous maps inside EL.

A function $\alpha$ encodes a partial continuous map from functions to numbers defined by

$$
\alpha(\beta)=\alpha(\bar{\beta} n)-1,
$$

where $n$ is the unique number such that

$$
\alpha(\bar{\beta} n) \neq 0 \wedge \forall m<n(\alpha(\bar{\beta} m)=0) ;
$$

if there is no such $n$, then $\alpha(\beta)$ is undefined. We write $\alpha(\beta) \downarrow$ when $\alpha(\beta)$ is defined, and we write $\alpha(\beta) \uparrow$ when $\alpha(\beta)$ is undefined.

Similarly, $\alpha$ encodes a partial continuous map from functions to functions defined by

$$
\alpha \mid \beta=\lambda n . \alpha\left(\langle n\rangle^{\wedge} \beta\right)
$$

provided that $\alpha\left(\langle n\rangle^{\wedge} \beta\right) \downarrow$ for every $n$. We write $\alpha \mid \beta \downarrow$ when $\alpha \mid \beta$ is defined, and we write $\alpha \mid \beta \uparrow$ when $\alpha \mid \beta$ is undefined. We use the left associative convention for $\mid$, that is, we will write $\alpha|\beta| \gamma$ for $(\alpha \mid \beta) \mid \gamma$. Consequently, $\alpha|\beta| \gamma \downarrow$ abbreviates $\alpha|\beta \downarrow \wedge(\alpha \mid \beta)| \gamma \downarrow$, and so on.

Every partial continuous map $F$ from functions to functions whose domain is a $G_{\delta}$-set admits a representation of the form $F(\xi)=\varphi \mid \xi$. We will write $\Lambda \xi . F(\xi)$ for a function $\varphi$ that represents $F$ in this way. There are always multiple choices for $\varphi$, but in all instances of this fact that we will use there is a natural choice of $\varphi$ that can be read from the description of $F$.

2.2 Compact sets of functions in EL Our results of Section 4 depend on the Lifschitz variant of realizability with functions due to van Oosten. To formalize this notion of realizability, we need to introduce an encoding of compact sets of functions.

Every function $\alpha$ encodes a compact set of functions defined by

$$
[\alpha]=\left\{\xi \leq \alpha \pi_{0}: \alpha \pi_{1}(\xi) \uparrow\right\} .
$$


Formally, we think of $\xi \in[\alpha]$ as an abbreviation for the statement

$$
\forall n\left(\xi(n) \leq \alpha \pi_{0}(n) \wedge \alpha \pi_{1}(\bar{\xi} n)=0\right) .
$$

We will write $[\alpha] \neq \varnothing$ to assert that $[\alpha]$ is inhabited: $\exists \xi(\xi \in[\alpha])$.

For a sound theory of compact sets, we will make frequent use of the weak König lemma:

$$
T(\alpha, \beta) \wedge \forall n \exists x(|x|=n \wedge \alpha(x)=0) \rightarrow \exists \xi \leq \beta \forall n(\alpha(\bar{\xi} n)=0),
$$

where $T(\alpha, \beta)$ says that $\{x: \alpha(x)=0\}$ is a tree bounded by $\beta$ :

$$
\forall x, y\left(\alpha\left(x^{\wedge}\langle y\rangle\right)=0 \rightarrow \alpha(x)=0 \wedge y \leq \beta(|x|)\right) .
$$

With this axiom, the statement $[\alpha] \neq \varnothing$ is equivalent to a $\Pi_{1}^{0}$-formula.

Van Oosten [10] shows that many properties of compact sets can be formalized in the theory $E L+W K L+M$. In particular, [10, Lemma 5.7] will be useful.

Lemma 2.1 There is a function term ı such that $\mathrm{EL}+\mathrm{WKL}+\mathrm{M}$ proves that

$$
\forall \xi \in[\alpha](\varphi \mid \xi \downarrow) \rightarrow \iota \mid\langle\varphi, \alpha\rangle \downarrow \wedge \forall \zeta(\zeta \in[\iota \mid\langle\varphi, \alpha\rangle] \leftrightarrow \exists \xi \in[\alpha](\zeta=\varphi \mid \xi)) .
$$

In other words, if $[\alpha] \subseteq \operatorname{dom} \varphi$, then $[\iota \mid\langle\varphi, \alpha\rangle]=\{\varphi \mid \xi: \xi \in[\alpha]\}$.

It is unclear whether the Markov principle $M$ is necessary to establish this and other lemmas from [10].

\section{Classical Consequences of GC}

Troelstra's generalized continuity principle is the scheme

$$
\forall \xi(B(\xi) \rightarrow \exists \zeta A(\xi, \zeta)) \rightarrow \exists \alpha \forall \xi(B(\xi) \rightarrow \alpha \mid \xi \downarrow \wedge A(\xi, \alpha \mid \xi)),
$$

where $B(\xi)$ is in $\mathrm{N}_{K}$ (defined below) and $A(\xi, \zeta)$ is arbitrary. One immediate consequence of $\mathrm{GC}$ is that if $A(\xi, \zeta)$ defines the graph of a total function, then this function must be continuous. It follows that GC is plainly false in the classical system RCA.

However, we will momentarily define two classes of formulas $\mathrm{N}_{K}$ and $\Gamma_{K}$ such that consequences of GC of the form

$$
\forall \xi(B(\xi) \rightarrow \exists \zeta A(\xi, \zeta)),
$$

where $B(\xi)$ is in $\mathrm{N}_{K}$ and $A(\xi, \zeta)$ is in $\Gamma_{K}$ are not only consequences of RCA, but the sequential form

$$
\forall \xi\left(\forall n B\left(\xi_{n}\right) \rightarrow \exists \zeta \forall n A\left(\xi_{n}, \zeta_{n}\right)\right)
$$

is also a consequence of RCA.

The proof of this fact relies on Kleene's realizability with functions (see Kleene and Vesley [5]), which is defined next.

Definition 3.1 We have the following:

- $\alpha \operatorname{rf} A$ is $A$ for atomic $A$,

- $\alpha \operatorname{rf}(A \wedge B)$ is $\alpha \pi_{0}$ rf $A \wedge \alpha \pi_{1}$ rf $B$,

- $\alpha \operatorname{rf}(A \rightarrow B)$ is $\forall \xi(\beta$ rf $A \rightarrow \alpha|\xi \downarrow \wedge \alpha| \xi \operatorname{rf} B)$,

- $\alpha$ rf $\forall x A$ is $\forall x\left(\alpha_{x}\right.$ rf $\left.A\right)$,

- $\alpha \operatorname{rf} \forall \xi A$ is $\forall \xi(\alpha|\xi \downarrow \wedge \alpha| \xi \operatorname{rf} A)$,

- $\alpha \operatorname{rf} \exists x A$ is $\alpha \sigma \operatorname{rf} A[x / \alpha(0)]$,

- $\alpha \operatorname{rf} \exists \xi A$ is $\alpha \pi_{1} \operatorname{rf} A\left[\xi / \alpha \pi_{0}\right]$. 
Note that $\alpha$ rf $A$ never involves existential quantifiers, except to say that $\alpha \mid \xi \downarrow$, in which case the scope of the existential quantifier is quantifier-free. It follows that $\alpha$ rf $A$ always belongs to the class $\mathrm{N}_{K}$.

\section{Definition 3.2}

- If $A$ is quantifier-free, then $A, \exists x A$, and $\exists \xi A$ are in $\mathrm{N}_{K}$.

- If $A, B$ are in $\mathrm{N}_{K}$, then so are $A \wedge B, A \rightarrow B, \forall x A$, and $\forall \xi A$.

In fact, the formulas of $\mathrm{N}_{K}$ are precisely the formulas which realize themselves in the sense of [9, Lemma 3.3.8].

Lemma 3.3 If $B(\xi) \in \mathrm{N}_{K}$, then

$$
\mathrm{EL} \vdash \exists \alpha(\alpha \operatorname{rf} B(\xi)) \leftrightarrow B(\xi) .
$$

In fact, there is a function term $\omega_{B}$ such that

$$
\mathrm{EL} \vdash B(\xi) \leftrightarrow \omega_{B}\left|\xi \downarrow \wedge \omega_{B}\right| \xi \operatorname{rf} B(\xi) .
$$

Stated in full generality, $B$ could depend on more than one argument (and hence so would $\omega_{B}$ ). However, this more general statement can be derived from Lemma 3.3 by packing all the arguments into one.

Kleene's realizability with functions was given the following characterization by Troelstra [9, Theorem 3.3.11].

Theorem 3.4 (Characterization of $\mathrm{r} \mathrm{f}$ ) For every formula $A$,

(a) $\mathrm{EL}+\mathrm{GC} \vdash A \leftrightarrow \exists \alpha(\alpha \operatorname{rf} A)$,

(b) $\mathrm{EL}+\mathrm{GC} \vdash A \Leftrightarrow \mathrm{EL} \vdash \exists \alpha(\alpha \operatorname{rf} A)$.

If $A$ has the property that EL $\vdash \exists \alpha(\alpha \operatorname{rf} A) \rightarrow A$, then we have

$$
\mathrm{EL}+\mathrm{GC} \vdash A \Leftrightarrow \mathrm{EL} \vdash A .
$$

Thus EL + GC is conservative over EL for formulas with this property. Lemma 3.3 shows that every formula in $\mathrm{N}_{K}$ has this property, but so do many other formulas.

\section{Definition 3.5}

- Quantifier-free formulas are in $\Gamma_{K}$.

- If $A, B$ are in $\Gamma_{K}$, then so are $A \wedge B, \forall x A, \forall \xi A, \exists x A$, and $\exists \xi A$.

- If $A$ is in $\mathrm{N}_{K}$ and $B$ is in $\Gamma_{K}$, then $A \rightarrow B$ is in $\Gamma_{K}$.

The following fact is implicit in [9, Theorem 3.6.18].

Lemma 3.6 If $A \in \Gamma_{K}$, then $\mathrm{EL} \vdash \exists \alpha(\alpha$ rf $A) \rightarrow A$.

Thus, by the characterization of $r f$, it follows that GC is conservative over EL for formulas in $\Gamma_{K}$.

Together, the above results imply the following.

Proposition 3.7 Suppose that $B(\xi) \in \mathrm{N}_{K}$ and that $A(\xi, \zeta) \in \Gamma_{K}$. If

$$
\mathrm{EL}+\mathrm{GC} \vdash \forall \xi(B(\xi) \rightarrow \exists \zeta A(\xi, \zeta)),
$$

then

$$
\mathrm{EL} \vdash \exists \alpha \forall \xi(B(\xi) \rightarrow \alpha \mid \xi \downarrow \wedge A(\xi, \alpha \mid \xi))
$$


Proof By Theorem 3.4, we know that

$$
\mathrm{EL} \vdash \exists \beta(\beta \text { rf } \forall \xi(B(\xi) \rightarrow \exists \zeta A(\xi, \zeta))) .
$$

Work in EL, and assume that $\beta$ rf $\forall \xi(B(\xi) \rightarrow \exists \zeta A(\xi, \zeta))$. Unpacking Definition 3.1 , we see that

$$
\gamma \operatorname{rf} B(\xi) \rightarrow \beta|\xi| \gamma \downarrow \wedge(\beta|\xi| \gamma) \pi_{1} \operatorname{rf} A\left(\xi,(\beta|\xi| \gamma) \pi_{0}\right) .
$$

Since $B(\xi) \in \mathrm{N}_{K}$, it follows from Lemma 3.3 that there is a term $\omega_{B}$ such that $B(\xi) \leftrightarrow \omega_{B}\left|\xi \downarrow \wedge \omega_{B}\right| \xi \operatorname{rf} B(\xi)$. Finally, since $A(\xi, \zeta) \in \Gamma_{K}$, it follows from Lemma 3.6 that $\alpha=\Lambda \xi .\left(\beta|\xi|\left(\omega_{B} \mid \xi\right)\right) \pi_{0}$, as required.

By the deduction theorem, the above result also holds when EL is replaced by $E L+$ $\Delta$, where $\Delta$ is any collection of sentences from $\mathrm{N}_{K}$.

Definition 3.8 Let $\mathrm{CN}$ be the set of all sentences $A$ from $\mathrm{N}_{K}$ such that RCA $\vdash A$. In other words, $\mathrm{CN}$ consists of all consequences of the law of excluded middle which belong to the syntactic class $\mathrm{N}_{K}$.

Note that CN includes the Markov principle $\mathrm{M}$.

Our uniformization result for this section is the following.

Corollary 3.9 Suppose that $B(\xi)$ is from $\mathrm{N}_{K}$ and that $A(\xi, \zeta)$ is from $\Gamma_{K}$. If

$$
\mathrm{EL}+\mathrm{GC}+\mathrm{CN} \vdash \forall \xi(B(\xi) \rightarrow \exists \zeta A(\xi, \zeta)),
$$

then

$$
\mathrm{RCA} \vdash \forall \xi\left(\forall n B\left(\xi_{n}\right) \rightarrow \exists \zeta \forall n A\left(\xi_{n}, \zeta_{n}\right)\right) .
$$

Proof Suppose that

$$
\mathrm{EL}+\mathrm{GC}+\mathrm{CN} \vdash \forall \xi(B(\xi) \rightarrow \exists \zeta A(\xi, \zeta)) .
$$

By Lemma 3.7, we know that

$$
\mathrm{EL}+\mathrm{CN} \vdash \exists \alpha \forall \xi(B(\xi) \rightarrow \alpha \mid \xi \downarrow \wedge A(\xi, \alpha \mid \xi)) .
$$

Now work in RCA, which extends EL $+\mathrm{CN}$. Given $\alpha$ such that

$$
\forall \xi(B(\xi) \rightarrow \alpha \mid \xi \downarrow \wedge A(\xi, \alpha \mid \xi)),
$$

if $\xi$ is such that $\forall n B\left(\xi_{n}\right)$, then $\zeta=\left\langle\alpha \mid \xi_{n}\right\rangle_{n=0}^{\infty}$ is such that $\forall n A\left(\xi_{n}, \zeta_{n}\right)$. It follows that

$$
\mathrm{RCA} \vdash \forall \xi\left(\forall n B\left(\xi_{n}\right) \rightarrow \exists \zeta \forall n A\left(\xi_{n}, \zeta_{n}\right)\right) .
$$

Note that this proof gives much more than the conclusion of the theorem requires. Indeed, Proposition 3.7 is a much stronger result than Corollary 3.9. Nevertheless, Corollary 3.9 has several uses, and its proof constitutes a nice warm-up for the next section.

Remark 3.10 In reverse mathematics, it is traditional to use the base system RCA $A_{0}$, which postulates only $\Sigma_{1}^{0}$-induction, rather than the system RCA, which postulates full induction. Unfortunately, following the proof-theoretic tradition, Troelstra assumes full induction throughout (see [9]). However, a close inspection of Troelstra's arguments shows that this assumption is not necessary to establish the characterization and conservation results for $r f$. Therefore, Proposition 3.7 and Corollary 3.9 have analogues with EL and RCA replaced by $E L_{0}$ and RCA $A_{0}$, respectively. 


\section{Classical Consequences of $\mathrm{GC}_{L}$}

Van Oosten's Lifschitz generalized continuity principle is the scheme

$$
\begin{aligned}
\forall \xi(B(\xi) \rightarrow \exists \zeta A(\xi, \zeta)) & \\
& \rightarrow \exists \alpha \forall \xi(B(\xi) \rightarrow \alpha \mid \xi \downarrow \wedge[\alpha \mid \xi] \neq \varnothing \wedge \forall \zeta \in[\alpha \mid \xi] A(\xi, \zeta)), \quad\left(\mathrm{GC}_{L}\right)
\end{aligned}
$$

where $B(\xi)$ is in $\mathrm{N}_{L}$ (defined below) and $A(\xi, \zeta)$ is arbitrary. Unlike GC, which offers a single witness for $\exists \zeta A(\xi, \zeta), \mathrm{GC}_{L}$ offers a nonempty compact set of witnesses for $\exists \zeta A(\xi, \zeta)$. The parameter for this compact set varies continuously with $\xi$, but there is no general way to continuously select a single element from this compact set. Thus, $\mathrm{GC}$ implies $\mathrm{GC}_{L}$, but the converse is false.

Nevertheless, $\mathrm{GC}_{L}$ still implies Brouwer's continuity theorem. Indeed, if $A(\xi, \zeta)$ describes the graph of a total function, then the compact set of witnesses produced by $\mathrm{GC}_{L}$ must be a singleton set. Since it is possible to continuously extract the unique element of a singleton set from its parameter (see [10, Lemma 5.3]), this shows that $A(\xi, \zeta)$ describes the graph of a continuous function. Like $\mathrm{GC}$, it follows that $\mathrm{GC}_{L}$ is also classically false.

Similarly to the case of GC, we will define two classes of formulas $\mathrm{N}_{L}$ and $\Gamma_{L}$ such that consequences of $\mathrm{EL}+\mathrm{WKL}+\mathrm{M}+\mathrm{GC}_{L}$ of the form

$$
\forall \xi(B(\xi) \rightarrow \exists \zeta A(\xi, \zeta)),
$$

where $B(\xi)$ is in $\mathrm{N}_{L}$ and $A(\xi, \zeta)$ is in $\Gamma_{L}$, are not only consequences of RCA + WKL, but the sequential form

$$
\forall \xi\left(\forall n B\left(\xi_{n}\right) \rightarrow \exists \zeta \forall n A\left(\xi_{n}, \zeta_{n}\right)\right)
$$

is also a consequence of RCA + WKL. The proof of this fact relies on Lifschitz realizability with functions, which was introduced by van Oosten [10].

Definition 4.1 We have the following:

- $\alpha \operatorname{lrf} A$ is $A$ for atomic $A$,

- $\alpha \operatorname{lrf}(A \wedge B)$ is $\alpha \pi_{0} \operatorname{lrf} A \wedge \alpha \pi_{1} \operatorname{lrf} B$,

- $\alpha \operatorname{lrf}(A \rightarrow B)$ is $\forall \beta(\beta \operatorname{lrf} A \rightarrow \alpha|\beta \downarrow \wedge \alpha| \beta \operatorname{lrf} B)$,

- $\alpha \operatorname{lrf} \forall x A$ is $\forall x\left(\alpha_{x} \operatorname{lrf} A\right)$,

- $\alpha \operatorname{lrf} \forall \xi A$ is $\forall \xi(\alpha|\xi \downarrow \wedge \alpha| \xi \operatorname{lrf} A)$,

- $\alpha \operatorname{lrf} \exists x A$ is $[\alpha] \neq \varnothing \wedge \forall \beta \in[\alpha](\beta \sigma \operatorname{lrf} A[x / \beta(0)])$,

- $\alpha \operatorname{lrf} \exists \xi A$ is $[\alpha] \neq \varnothing \wedge \forall \beta \in[\alpha]\left(\beta \pi_{1} \operatorname{lrf} A\left[\xi / \beta \pi_{0}\right]\right)$.

The analogue of the class $\mathrm{N}_{K}$ is the broader class $\mathrm{N}_{L}$.

\section{Definition 4.2}

- If $A$ is quantifier-free, then $A, \exists x A, \exists \xi A$ are in $\mathrm{N}_{L}$.

- If $A$ is quantifier-free and $\tau$ is a function term in which $\xi$ does not occur, then $\exists \xi \leq \tau \forall z A$ is in $\mathrm{N}_{L}$. Similarly, if $A$ is quantifier-free and $t$ is a number term in which $x$ does not occur, then $\exists x \leq t \forall z A$ is in $\mathrm{N}_{L}$.

- If $A, B$ are in $\mathrm{N}_{L}$, then so are $A \wedge B, A \rightarrow B, \forall x A$, and $\forall \xi A$.

With the aid of the second clause, the disjunction of one or more $\Pi_{1}^{0}$-statements can be formulated in $\mathrm{N}_{L}$. Thus, statements like the dichotomy law for Cauchy real numbers (discussed in Section 5) can be expressed in $\mathrm{N}_{L}$ but not in $\mathrm{N}_{K}$. 
Again, the formula $\alpha \operatorname{lrf} A$ is always in $\mathrm{N}_{L}$. In fact, the formulas of $\mathrm{N}_{L}$ are precisely the formulas which realize themselves in the following sense (see [10, Lemma 5.12]).

Lemma 4.3 If $B(\xi) \in \mathrm{N}_{L}$, then

$$
\mathrm{EL}+\mathrm{WKL}+\mathrm{M} \vdash \exists \alpha(\alpha \operatorname{lrf} B(\xi)) \leftrightarrow B(\xi) .
$$

In fact, there is a function term $\omega_{B}$ such that

$$
\mathrm{EL}+\mathrm{WKL}+\mathrm{M} \vdash B(\xi) \leftrightarrow \omega_{B}\left|\xi \downarrow \wedge \omega_{B}\right| \xi \operatorname{lrf} B(\xi) .
$$

Again, there is a more general form of this which allows $B$ to have more than one parameter, but this can be derived from the above by packing all arguments into one.

Lifschitz realizability with functions was characterized by van Oosten [10, Theorem 5.15].

Theorem 4.4 (Characterization of $\ln \mathrm{f}$ ) For every formula $A$,

(a) $\mathrm{EL}+\mathrm{WKL}+\mathrm{M}+\mathrm{GC}_{L} \vdash A \leftrightarrow \exists \alpha(\alpha \operatorname{lrf} A)$,

(b) $\mathrm{EL}+\mathrm{WKL}+\mathrm{M}+\mathrm{GC}_{L} \vdash A \Leftrightarrow \mathrm{EL}+\mathrm{WKL}+\mathrm{M} \vdash \exists \alpha(\alpha \operatorname{lrf} A)$.

Again, it is unclear whether $M$ is necessary for this characterization of $\operatorname{lr} f$.

The class $\Gamma_{L}$ is defined as follows.

\section{Definition 4.5}

- Quantifier-free formulas are in $\Gamma_{L}$.

- If $A, B$ are in $\Gamma_{L}$, then so are $A \wedge B, \forall x A, \forall \xi A, \exists x A$, and $\exists \xi A$.

- If $A$ is in $\mathrm{N}_{L}$ and $B$ is in $\Gamma_{L}$, then $A \rightarrow B$ is in $\Gamma_{L}$.

Together with the characterization of $\operatorname{lrf}$, the following fact shows that $\mathrm{GC}_{L}$ is conservative over $E L+W K L+M$ for formulas in $\Gamma_{L}$.

Lemma 4.6 If $A \in \Gamma_{L}$, then $\mathrm{EL}+\mathrm{WKL}+\mathrm{M} \vdash \exists \alpha(\alpha \operatorname{lrf} A) \rightarrow A$.

Proof sketch The proof of this lemma is a straightforward induction on the complexity of $A$. We prove only the implication case.

Work in $\mathrm{EL}+\mathrm{WKL}+\mathrm{M}$. Suppose that $\alpha \operatorname{lrf}(B \rightarrow A)$, where $A$ is from $\Gamma_{L}$ and $B$ is from $\mathrm{N}_{L}$. We need to show that $B \rightarrow A$. By definition of $\operatorname{lr} \mathrm{f}$, we then have that if $\beta \operatorname{lrf} B$, then $\alpha \mid \beta \downarrow$ and $\alpha \mid \beta \operatorname{lrf} A$. Assume $B$. By Lemma 4.3, there is a function term $\omega$ such that $\omega \operatorname{lrf} B$. It follows that $\alpha \mid \omega \downarrow$ and $\alpha \mid \omega \operatorname{lrf} A$. Therefore $A$, by the induction hypothesis.

Together, the above results imply the following.

Proposition 4.7 Suppose that $B(\xi) \in \mathrm{N}_{L}$ and that $A(\xi, \zeta) \in \Gamma_{L}$. If

$$
\mathrm{EL}+\mathrm{WKL}+\mathrm{M}+\mathrm{GC}_{L} \vdash \forall \xi(B(\xi) \rightarrow \exists \zeta A(\xi, \zeta))
$$

then

$$
\mathrm{EL}+\mathrm{WKL}+\mathrm{M} \vdash \exists \alpha \forall \xi(B(\xi) \rightarrow \alpha \mid \xi \downarrow \wedge[\alpha \mid \xi] \neq \varnothing \wedge \forall \zeta \in[\alpha \mid \xi] A(\xi, \zeta)) .
$$

Proof By Theorem 4.4, we know that

$$
\mathrm{EL}+\mathrm{WKL}+\mathrm{M} \vdash \exists \beta(\beta \operatorname{lrf} \forall \xi(B(\xi) \rightarrow \exists \zeta A(\xi, \zeta))) .
$$

Work in $\mathrm{EL}+\mathrm{WKL}+\mathrm{M}$, and assume that $\beta \operatorname{lrf} \forall \xi(B(\xi) \rightarrow \exists \zeta A(\xi, \zeta))$. Unpacking the definition of $\operatorname{lrf}$, we see that if $\gamma \operatorname{lrf} B(\xi)$, then $\beta|\xi| \gamma \downarrow,[\beta|\xi| \gamma] \neq \varnothing$, and

$$
\forall \zeta \in[\beta|\xi| \gamma]\left(\zeta \pi_{1} \operatorname{lrf} A\left(\xi, \zeta \pi_{0}\right)\right)
$$


Since $B(\xi) \in \mathrm{N}_{L}$, it follows from Lemma 4.3 that there is a term $\omega_{B}$ such that

$$
B(\xi) \leftrightarrow \omega_{B}\left|\xi \downarrow \wedge \omega_{B}\right| \xi \operatorname{lrf} B(\xi) .
$$

Finally, since $A(\xi, \zeta) \in \Gamma_{L}$, it follows from Lemma 4.6 that

$$
\alpha=\Lambda \xi . \iota \mid\left\langle\Lambda \zeta . \zeta \pi_{0}, \beta|\xi|\left(\omega_{B} \mid \xi\right)\right\rangle
$$

is as required, where $\iota$ is as in Lemma 2.1.

As for Proposition 3.7, we can add to the theories in Proposition 4.7 any collection of sentences from $\mathrm{N}_{L}$.

Definition 4.8 Let $\mathrm{CN}_{L}$ be the collection of all sentences $A$ from $\mathrm{N}_{L}$ such that $\mathrm{RCA}+\mathrm{WKL} \vdash A$. In other words, $\mathrm{CN}_{L}$ consists of all consequences of the law of excluded middle which belong to the syntactic class $\mathrm{N}_{L}$.

Note that $\mathrm{CN}_{L}$ includes the Markov principle $\mathrm{M}$ as well as the lesser limited principle of omniscience (LLPO) (see Section 5).

Corollary 4.9 Suppose that $B(\xi)$ is from $\mathrm{N}_{L}$ and that $A(\xi, \zeta)$ is from $\Gamma_{L}$. If

$$
\mathrm{EL}+\mathrm{WKL}+\mathrm{GC}_{L}+\mathrm{CN}_{L} \vdash \forall \xi(B(\xi) \rightarrow \exists \zeta A(\xi, \zeta))
$$

then

$$
\mathrm{RCA}+\mathrm{WKL} \vdash \forall \xi\left(\forall n B\left(\xi_{n}\right) \rightarrow \exists \zeta \forall n A\left(\xi_{n}, \zeta_{n}\right)\right) .
$$

Proof Suppose that

$$
\mathrm{EL}+\mathrm{WKL}+\mathrm{GC}_{L}+\mathrm{CN}_{L} \vdash \forall \xi(B(\xi) \rightarrow \exists \zeta A(\xi, \zeta)) .
$$

By Proposition 4.7, we know that

$$
\mathrm{EL}+\mathrm{WKL}+\mathrm{CN}_{L} \vdash \exists \alpha \forall \xi(B(\xi) \rightarrow \alpha \mid \xi \downarrow \wedge[\alpha \mid \xi] \neq \varnothing \wedge \forall \zeta \in[\alpha \mid \xi] A(\xi, \zeta))
$$

Now work in RCA + WKL, which extends $E L+W K L+C_{L}$. Find $\alpha$ such that if $B(\xi)$, then

$$
\alpha \mid \xi \downarrow \wedge[\alpha \mid \xi] \neq \varnothing \wedge \forall \zeta \in[\alpha \mid \xi] A(\xi, \zeta) .
$$

If $\xi$ is such that $\forall n B\left(\xi_{n}\right)$, then

$$
\forall n\left(\alpha \mid \xi_{n} \downarrow \wedge\left[\alpha \mid \xi_{n}\right] \neq \varnothing\right) .
$$

By [8, Lemma VIII.2.4], we can find a $\zeta$ such that $\zeta_{n} \in\left[\alpha \mid \xi_{n}\right]$ for every $n$. It then follows that $\forall n A\left(\xi_{n}, \zeta_{n}\right)$. We have just shown that

$$
\mathrm{RCA}+\mathrm{WKL} \vdash \forall \xi\left(\forall n B\left(\xi_{n}\right) \rightarrow \exists \zeta \forall n A\left(\xi_{n}, \zeta_{n}\right)\right) .
$$

Remark 4.10 As in Remark 3.10, it would be desirable to eliminate the induction assumptions from Corollary 4.9. Unfortunately, van Oosten's arguments from [10] do appear to make some use of this inductive assumption. Close inspection reveals that these uses are limited to $\Pi_{1}^{0}$-bounding; therefore, Corollary 4.9 does have an analogue with RCA + WKL replaced by $R C A_{0}+W K L+B \Pi_{1}^{0}$. 


\section{Applications}

To compare the earlier results of Hirst and Mummert with ours, it is useful to compare the syntactic restrictions involved, specifically [4, Theorem 3.6], since the syntactic conditions for [4, Theorem 5.6] are even more restrictive.

The analogue of $\mathrm{N}_{K}$ and $\mathrm{N}_{L}$ for Hirst and Mummert are $\exists$-free formulas: formulas built in the usual manner but without the use of existential quantifiers or disjunctions. The $\exists$-free formulas are a proper subset of $\mathrm{N}_{K}$ and hence $\mathrm{N}_{L}$ since some existential quantifiers are allowed by the first clause of Definition 3.2, and still more are allowed by the second clause of Definition 4.2.

The analogue of $\Gamma_{K}$ and $\Gamma_{L}$ for Hirst and Mummert is the class $\Gamma_{1}$, which is defined in exactly the same way, except that hypotheses of conditionals are restricted to $\exists$-free formulas. Thus, $\Gamma_{1}$ is also a proper subset of $\Gamma_{K}$ and hence $\Gamma_{L}$.

To illustrate the difference, consider this familiar statement:

Every $(n \times n)$-matrix with nonzero determinant has an inverse.

The "nonzero determinant" hypothesis is not expressible by an $\exists$-free formula, since to say that a Cauchy real or complex number (see below) is apart from zero requires an existential quantifier. However, this hypothesis is expressible in $\mathrm{N}_{K}$. Since $\mathrm{EL}$ proves that every $(n \times n)$-matrix with nonzero determinant has an inverse, it follows that the sequential form of the above statement is provable in RCA. The reader should not feel too enlightened by this simple example since the obvious proof is nothing more than Cramer's rule.

On the other hand, the results of Hirst and Mummert allow for higher types, while ours only involve first-order and second-order types. Therefore, there is a vast sea of statements for which the results of Hirst and Mummert apply but ours do not. Still, the nonprovability examples that Hirst and Mummert give are all second-order, so they all have equivalents in our context. In particular, neither $\mathrm{EL}+\mathrm{GC}+\mathrm{CN}$ nor $\mathrm{EL}+\mathrm{WKL}+\mathrm{GC}_{L}+\mathrm{CN}_{L}$ prove that every $(n \times n)$-matrix has a Jordan canonical form.

5.1 Trichotomy and dichotomy for Cauchy reals A Cauchy real is a rational-valued function $\alpha$ such that $|\alpha(s)-\alpha(t)| \leq 2^{-s}$ for all $s<t$. We write $\alpha \in \mathbb{R}^{C}$ to abbreviate the statement that $\alpha$ is a Cauchy real. If $\alpha, \beta \in \mathbb{R}^{C}$, then we define

$$
\alpha=\beta \leftrightarrow \forall s\left(|\alpha(s)-\beta(s)| \leq 2^{1-s}\right) .
$$

We also define

$$
\alpha>\beta \leftrightarrow \exists s\left(\alpha(s)-\beta(s)>2^{1-s}\right)
$$

and

$$
\alpha \leq \beta \leftrightarrow \forall s\left(\alpha(s)-\beta(s) \leq 2^{1-s}\right) .
$$

Note that $\alpha \leq \beta \leftrightarrow \neg(\alpha>\beta)$ and $\alpha>\beta \rightarrow \neg(\alpha \leq \beta)$, but the implication $\neg(\alpha \leq \beta) \rightarrow \alpha>\beta$ is equivalent to the Markov principle M.

The trichotomy law

$$
\alpha<\beta \vee \alpha=\beta \vee \alpha>\beta
$$

and the formally weaker dichotomy law

$$
\alpha \leq \beta \vee \alpha \geq \beta
$$


are both consequences of the law of excluded middle. However, over $E L_{0}$ these are respectively equivalent to the limited principle of omniscience

$$
\exists n(\xi(n) \neq 0) \vee \forall n(\xi(n)=0)
$$

and the lesser limited principle of omniscience

$$
\neg(\exists n(\xi(n) \neq 0) \wedge \exists n(\zeta(n) \neq 0)) \rightarrow \forall n(\xi(n)=0) \vee \forall n(\zeta(n)=0)
$$

(see [2] for details).

Proposition 5.1 The following equivalent statements are both provable in $\mathrm{RCA}$, but neither is provable in $\mathrm{EL}+\mathrm{GC}+\mathrm{CN}$ :

(a) the dichotomy law for Cauchy reals,

(b) the lesser limited principle of omniscience.

Proof The dichotomy law can be stated as

$$
\forall \alpha, \beta\left(\alpha, \beta \in \mathbb{R}^{C} \rightarrow \exists y((y=0 \rightarrow \alpha \leq \beta) \wedge(y \neq 0 \rightarrow \alpha \geq \beta))\right) .
$$

Inspection shows that this is in the form required for Corollary 3.9. Dorais, Hirst, and Shafer [2] have shown that the corresponding sequential form is equivalent to WKL over RCA $\mathrm{R}_{0}$; it follows that the dichotomy law is not provable in $E L+G C+C N$.

Proposition 5.2 The following equivalent statements are both provable in RCA, but neither is provable in $\mathrm{EL}+\mathrm{WKL}+\mathrm{GC}_{L}+\mathrm{CN}_{L}$ :

(a) the trichotomy law for Cauchy reals,

(b) the limited principle of omniscience.

Proof For all $\alpha, \beta \in \mathbb{R}^{C}$, the trichotomy law can be stated as

$$
\exists y((y=0 \rightarrow \alpha<\beta) \wedge(y=1 \rightarrow \alpha>\beta) \wedge(y>1 \rightarrow \alpha=\beta)) .
$$

This statement is in the form required for Corollary 4.9. Dorais, Hirst, and Shafer [2] have shown that the corresponding sequential form is equivalent to arithmetic comprehension over $\mathrm{RCA}_{0}$; it follows that the trichotomy law is not provable in $\mathrm{EL}+\mathrm{WKL}+\mathrm{GC}_{L}+\mathrm{CN}_{L}$.

5.2 Dedekind reals and Cauchy reals A Dedekind real is a decidable set $\delta$ of rationals such that

$$
\exists p, q \in \mathbb{Q}(p \in \delta \wedge q \notin \delta) \wedge \forall p, q \in \mathbb{Q}(p \in \delta \wedge q \notin \delta \rightarrow p<q) .
$$

We write $\delta \in \mathbb{R}^{D}$ to abbreviate the fact that $\delta$ is a Dedekind real. We say that a Cauchy real $\alpha$ and a Dedekind real $\delta$ are equivalent when

$$
\forall s \in \mathbb{N} \forall p, q \in \mathbb{Q}\left(p \in \delta \wedge q \notin \delta \rightarrow \neg\left(\alpha(s)+2^{1-s}<p \vee q<\alpha(s)-2^{1-s}\right)\right) .
$$

\section{Proposition 5.3}

(a) $\mathrm{EL}_{0}$ proves that every Dedekind real has an equivalent Cauchy real.

(b) $\mathrm{EL}_{0}+\mathrm{WKL}$ proves that every Cauchy real has an equivalent Dedekind real.

Proof The proof of part (a) is straightforward, so we only prove part (b). 
Suppose that $\alpha$ is a Cauchy real. Fix an enumeration $\left\langle q_{i}\right\rangle_{i=0}^{\infty}$ of $\mathbb{Q}$. Let $R(\alpha, x)$ denote the statement

$$
\begin{aligned}
\forall i< & |x|((x(i)=0 \vee x(i)=1) \\
& \wedge\left(\exists s \leq|x|\left(q_{i}<\alpha(s)-2^{1-s}\right) \rightarrow x(i)=1\right) \\
& \wedge\left(\exists s \leq|x|\left(q_{i}>\alpha(s)+2^{1-s}\right) \rightarrow x(i)=0\right) .
\end{aligned}
$$

Then the decidable set $\delta=\left\{q_{i}: \xi(i)=1\right\}$ is a Dedekind real equivalent to $\alpha$ if and only if $\forall n R(\alpha, \bar{\xi} n)$. Since $\forall n \exists x(|x|=n \wedge R(\alpha, x))$, it follows from WKL that there is a Dedekind real $\delta$ which is equivalent to $\alpha$.

Of course, $\mathrm{RCA}_{0}$ proves that every Cauchy real has an equivalent Dedekind real. However, the usual proof of this fact is nonuniform since it relies on first deciding whether or not the Cauchy real represents a rational number. Such lack of uniformity is actually necessary, as the next proposition shows.

Proposition 5.4 The system $\mathrm{EL}+\mathrm{GC}+\mathrm{CN}$ does not prove that every Cauchy real has an equivalent Dedekind real.

Proof Formally, the statement that every Cauchy real has an equivalent Dedekind real is as follows: for every $\alpha \in \mathbb{R}^{C}$ there is a $\delta \in \mathbb{R}^{D}$ such that

$$
\forall p, q \in \mathbb{Q} \forall s \in \mathbb{N}\left(p \in \delta \wedge q \notin \delta \rightarrow \neg\left(\alpha(s)+2^{1-s}<p \vee q<\alpha(s)-2^{1-s}\right)\right) .
$$

Inspection shows that this has the right form for Corollary 3.9. However, Hirst [3] has shown that the sequential form of this statement is equivalent to WKL over RCA $A_{0}$. It follows that the statement is not provable in $\mathrm{EL}+\mathrm{GC}+\mathrm{CN}$.

Note that dichotomy is trivially true for Dedekind reals (simply check in which half 0 is). Thus, Proposition 5.4 is actually a corollary of Proposition 5.1.

5.3 The fundamental theorem of algebra Cauchy complex numbers are pairs $\left\langle\xi_{0}, \xi_{1}\right\rangle$, where $\xi_{0}, \xi_{1} \in \mathbb{R}^{C}$. These are intended to represent the real and imaginary parts of the complex number. Thus, we write $\xi \in \mathbb{C}^{C}$ to abbreviate $\xi \pi_{0}, \xi \pi_{1} \in \mathbb{R}^{C}$. Addition and multiplication on complex numbers are defined as usual; it is not difficult to check that $\mathrm{EL}_{0}$ proves that $\mathbb{C}^{C}$ is a field. However, $\mathrm{EL}+\mathrm{GC}$ does not prove that $\mathbb{C}^{C}$ is algebraically complete.

Proposition 5.5 $\quad \mathrm{EL}+\mathrm{GC}+\mathrm{CN}$ does not prove that every complex number has a square root.

Proof Suppose, on the contrary, that EL + GC does prove that every complex number has a square root. It follows from Proposition 3.7 that EL proves the existence of some $\alpha$ such that

$$
(\forall \xi)\left(\xi \in \mathbb{C}^{C} \rightarrow \alpha|\xi \downarrow \wedge \alpha| \xi \in \mathbb{C}^{C} \wedge(\alpha \mid \xi)^{2}=\xi\right) .
$$

Since this statement is in $\Gamma_{K}$, it follows that EL proves the existence of such an $\alpha$. This is impossible since the axioms of EL are classically valid and there is no total continuous function on the complex numbers that selects one of the two square roots of its argument. 
The use of Proposition 3.7 instead of Corollary 3.9 was necessary for this argument since $\mathrm{RCA}_{0}$ does prove the sequential form

$$
\forall \xi\left(\forall n\left(\xi_{n} \in \mathbb{C}^{C}\right) \rightarrow \exists \zeta \forall n\left(\zeta_{n} \in \mathbb{C}^{C} \wedge \xi_{n}=\zeta_{n}^{2}\right)\right) .
$$

In particular, the converse of Corollary 3.9 is false.

While the fundamental theorem of algebra is not provable in $E L+G C+C N$, it is provable in $\mathrm{EL}+\mathrm{WKL}$.

Proposition 5.6 $\mathrm{EL}+\mathrm{WKL}$ proves that

$$
\forall \xi_{1}, \ldots, \xi_{n} \in \mathbb{C}^{C} \exists \zeta \in \mathbb{C}^{C}\left(\zeta^{n}+\xi_{1} \zeta^{n-1}+\cdots+\xi_{n}=0\right) .
$$

This is because EL proves that for any coefficients $\xi_{1}, \ldots, \xi_{n} \in \mathbb{C}^{C}$, there is a function $\alpha$ such that

$$
[\alpha]=\left\{\zeta \in \mathbb{C}^{C}: \zeta^{n}+\xi_{1} \zeta^{n-1}+\cdots+\xi_{n}=0\right\} .
$$

Then, by proving the existence of approximate roots, $E L+$ WKL proves that $[\alpha] \neq \varnothing$.

\section{Notes}

1. Note that in the reverse mathematics literature, WKL is normally used as an abbreviation for RCA together with the weak König lemma. We will avoid this practice since we also want to consider the weak König lemma in intuitionistic systems.

2. Elements of $\mathrm{N}_{K}$ are called "almost negative formulas" by Troelstra [9].

3. Elements of $\mathrm{N}_{L}$ are called "B $\Sigma_{2}^{1}$-negative formulas" by van Oosten.

4. Note that the statement of [10, Theorem 5.15(ii)] has a typo, which is corrected in our statement of Theorem 4.4(b).

\section{References}

[1] Brouwer, L. E. J., "Über Definitionsbereiche von-Funktionen," Mathematische Annalen, vol. 97 (1927), pp. 60-75. MR 1512354. DOI 10.1007/BF01447860. English translation in J. van Heijenoort, From Frege to Gödel: A Source Book in Mathematical Logic, 1879_ 1931, Harvard University Press, Cambridge, Mass., 1967, pp. 446-63. MR 0209111. 25

[2] Dorais, F. G., J. L. Hirst, and P. Shafer, "Reverse mathematics, trichotomy and dichotomy," Journal of Logic and Analysis, vol. 4 (2012), no. 13. MR 2955051. 36

[3] Hirst J. L., "Representations of reals in reverse mathematics," Bulletin of the Polish Academy of Sciences, Mathematics, vol. 55 (2007), pp. 303-16. Zbl 1138.03012. MR 2369116. DOI 10.4064/ba55-4-2. 37

[4] Hirst, J. L., and C. Mummert, "Reverse mathematics and uniformity in proofs without excluded middle," Notre Dame Journal of Formal Logic, vol. 52 (2011), pp. 149-62. Zbl 1225.03083. MR 2794648. DOI 10.1215/00294527-1306163. 26, 35

[5] Kleene, S. C., and R. E. Vesley, The Foundations of Intuitionistic Mathematics, Especially in Relation to Recursive Functions, North-Holland, Amsterdam, 1965. MR 0176922. 29

[6] Kohlenbach, U., "Higher order reverse mathematics," pp. 281-95 in Reverse Mathematics 2001, vol. 21 of Lecture Notes in Logic, Association for Symbolic Logic, La Jolla, Calif., 2005. MR 2185441. 26 
[7] Kohlenbach, U., Applied Proof Theory: Proof Interpretations and Their Use in Mathematics, Springer Monographs in Mathematics, Springer, Berlin, 2008. Zbl 1158.03002. MR 2445721. 26

[8] Simpson, S. G., Subsystems of Second Order Arithmetic, 2nd edition, Perspectives in Logic, Cambridge University Press, Cambridge; Association for Symbolic Logic, Poughkeepsie, N.Y., 2009. MR 2517689. DOI 10.1017/CBO9780511581007. 27, 34

[9] Troelstra, A. S., ed., Metamathematical Investigation of Intuitionistic Arithmetic and Analysis, vol. 344 of Lecture Notes in Mathematics, Springer, Berlin, 1973. Zbl 0275.02025. MR 0325352. 26, 27, 30, 31, 38

[10] van Oosten, J., "Lifschitz' realizability," Journal of Symbolic Logic, vol. 55 (1990), pp. 805-21. MR 1056390. DOI 10.2307/2274666. 26, 29, 32, 33, 34, 38

Department of Mathematics

Dartmouth College

Hanover, New Hampshire 03755

USA 